EPJ Web of Conferences 59, 03001 (2013)

DOI: $10.1051 /$ epjconf/20135903001

(C) Owned by the authors, published by EDP Sciences, 2013

\title{
Progress in the shock-ignition inertial confinement fusion concept
}

\author{
W. Theobald ${ }^{1, a}$, A. Casner ${ }^{2}$, R. Nora ${ }^{1}$, X. Ribeyre ${ }^{3}$, M. Lafon ${ }^{1,3}$, \\ K.S. Anderson ${ }^{1}$, R. Betti ${ }^{1,4}$, R.S. Craxton ${ }^{1}$, J.A. Delettrez ${ }^{1}$, J.A. Frenje ${ }^{5}$, \\ V.Yu. Glebov ${ }^{1}$, O.V. Gotchev ${ }^{1}$, M. Hohenberger ${ }^{1}$, S.X. Hu ${ }^{1}$, F.J. Marshall ${ }^{1}$, \\ R.L. McCrory ${ }^{1,4}$, D.D. Meyerhofer ${ }^{1,4}$, L.J. Perkins ${ }^{6}$, T.C. Sangster ${ }^{1}$, \\ G. Schurtz ${ }^{3}$, W. Seka ${ }^{1}$, V.A. Smalyuk ${ }^{1,6}$, C. Stoeckl ${ }^{1}$ and B. Yaakobi ${ }^{1}$ \\ ${ }^{1}$ Laboratory for Laser Energetics and Fusion Science Center, Rochester, NY, USA \\ ${ }^{2}$ CEA, DAM, DIF, Arpajon, France \\ ${ }^{3}$ Centre Lasers Intenses et Applications, University of Bordeaux, France \\ ${ }^{4}$ Departments of Mechanical Engineering and Physics, University of Rochester, Rochester, \\ NY, USA \\ ${ }^{5}$ Massachusetts Institute of Technology, Cambridge, MA, USA \\ ${ }^{6}$ Lawrence Livermore National Laboratory, Livermore, CA, USA
}

\begin{abstract}
Shock-ignition experiments with peak laser intensities of $\sim 8 \times 10^{15} \mathrm{~W} / \mathrm{cm}^{2}$ were performed. $\mathrm{D}_{2}$-filled plastic shells were compressed on a low adiabat by 40 of the 60 OMEGA beams. The remaining 20 beams were delayed and tightly focused onto the imploding shell to generate a strong shock. Up to $35 \%$ backscattering of laser energy was measured at the highest intensity. Hard x-ray measurements reveal a relatively low hot-electron temperature of $\sim 40 \mathrm{keV}$, independent of intensity and spike onset time.
\end{abstract}

Shock ignition (SI) is a two-step inertial confinement fusion concept in which a strong shock wave is launched at the end of the laser pulse to ignite the compressed core of a low-velocity implosion [1]. Two-step processes separate fuel assembly and ignition, relaxing driver requirements and promising high gains [1-3]. SI relies on highly shaped laser pulses that are within the pulse-shaping capabilities of currently operating laser systems like the National Ignition Facility (NIF) [4]. Proof-of-principle experiments can be carried out on the NIF [5]. This shows a promising route to high fusion gains at moderate laser energies. An intensity spike $\left(\sim 3 \times 10^{15}\right.$ to $\left.\sim 10^{16} \mathrm{~W} / \mathrm{cm}^{2}\right)$ at the end of the laser pulse launches the ignitor shock, and the final fuel assembly develops a centrally peaked pressure profile, lowering the ignition threshold compared to standard isobaric assemblies [6].

Parametric plasma instabilities [7] such as stimulated Brillouin scattering (SBS), stimulated Raman scattering (SRS), and two-plasmon-decay (TPD) instability are of concern in an ignition target design. The instabilities increase the back-reflection of the laser light from the target, degrading the laserenergy coupling to the capsule. They increase the fraction of the laser energy transferred to suprathermal electrons-a potential source of preheat that could reduce the final core compression. Significant laserplasma instabilities can be expected during the high-intensity ignitor spike. Whether or not these are

ae-mail: wthe@lle.rochester.edu

This is an Open Access article distributed under the terms of the Creative Commons Attribution License 2.0, which permits unrestricted use, distribution, and reproduction in any medium, provided the original work is properly cited. 

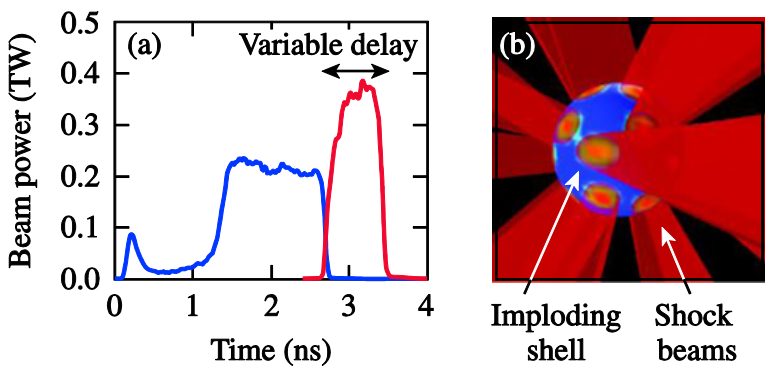

Figure 1. (a) The drive-pulse shape (blue) for the 40 beams and the high-intensity pulse (red) for the 20 spike beams. Beam delay and spike intensity was varied. (b) The 20 delayed spike beams were tightly focused onto the critical-density surface, where plasma instabilities led to the generation of energetic electrons and laser backscattering.

harmful depends on the hot-electron temperature and the amount of laser energy that is transferred into hot electrons at high laser intensities [8].

The objective of this work is to study the coupling of laser energy from high-intensity beams into a spherically imploding capsule and the laser-plasma instabilities at SI-relevant laser intensities. Previous SI experiments [9] on OMEGA [10] studied fuel assembly with 60-beam symmetric implosions. The shock wave was launched by a spike at the end of the pulse on all 60 beams with a maximum intensity of $\sim 7 \times 10^{14} \mathrm{~W} / \mathrm{cm}^{2}$. The intensity was too low to study laser-plasma instabilities for SI. Switching from a 60-beam to a $40+20$-beam configuration [11] on OMEGA using two separate pulse-shaping systems makes it possible to use tightly focused beams that generate a stronger shock wave than in the previous experiments.

The targets were 34 - to $36-\mu \mathrm{m}$-thick, $430-\mu \mathrm{m}$-outer-radius, deuterated plastic shells coated outside with a $0.1-\mu \mathrm{m}$ layer of aluminium and filled with $\mathrm{D}_{2}$ gas with a pressure of $\sim 25 \mathrm{~atm}$. The capsules were imploded using 40 of the 60 OMEGA beams with a low-adiabat pulse shape with $\sim 13.6 \mathrm{~kJ}$ of UV laser energy. The blue curve in Figure 1(a) shows the drive-pulse shape with a total duration of $2.7 \mathrm{~ns}$. The 351-nm-wavelength laser light was smoothed with polarization smoothing [12] and distributed phase plates (DPP's) [13]. A late shock was driven by the remaining 20 beams that were delayed and tightly focused to achieve intensities at the critical surface ranging from $\sim 0.8 \times 10^{15}$ to $\sim 8 \times 10^{15} \mathrm{~W} / \mathrm{cm}^{2}$. The 20 beams $(\sim 4.6 \mathrm{~kJ})$ used an $\sim 600$-ps FWHM square pulse shape (red curve) without polarization smoothing or DPP in most of the shots. In a few shots, small-spot, indirect-drive "IDI-300" DPP's were used [14]. The intensity was varied by shifting the focus of the 20 beams relative to the shell's center. The nominal laser intensity is quoted for the location of the critical-density plasma surface calculated by a one-dimensional (1-D) hydrodynamic simulation with the code LILAC [15]. Figure 1(b) shows a schematic for intermediate spike intensity. The foci of the 20 spike beams did not overlap at the critical density for most lens positions. Only at the lowest intensity of $\sim 0.8 \times 10^{15} \mathrm{~W} / \mathrm{cm}^{2}$, the 20 spike beams partially overlapped. The experimental observables are the neutron yield [16], the backscattered laser energy [17], the hard x-ray signal [18], and the neutron-rate-averaged areal density [19]. The hard x-ray signals were measured with four channels $(>20,>40,>60$, and $>80 \mathrm{keV})$ [18]. Areal densities $(\rho R)$ were inferred from secondary proton spectra [19].

Hydrodynamic simulations were performed with the code CHIC [2]. Figure 2 shows the calculated areal density as a function of the polar angle. The solid curve is from a 2-D simulation and the dashed line is from a 1-D simulation. The experimental values are noted by solid circles. The simulation values are higher because they represent the areal density at peak neutron-production, whereas the experimental values are averaged over the observed neutron production rate. The modulations may be dominated by the dodecahedron imprint pattern from the 20 spike beams. 


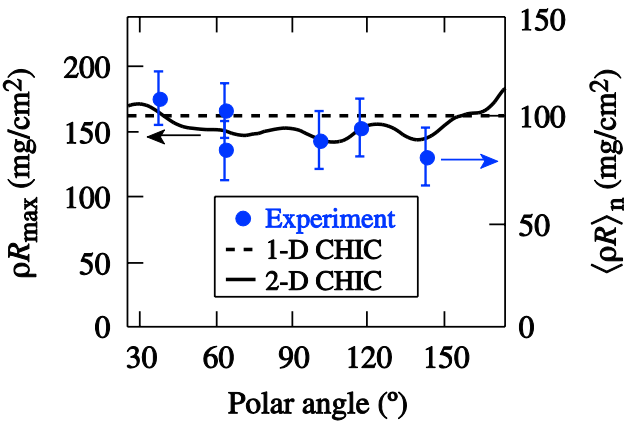

Figure 2. Simulated (curves) and measured areal density (circles) as a function of the polar angle. The dashed line is from a 1-D simulation and the solid curve from a 2-D hydrodynamic simulation with the code CHIC [2].
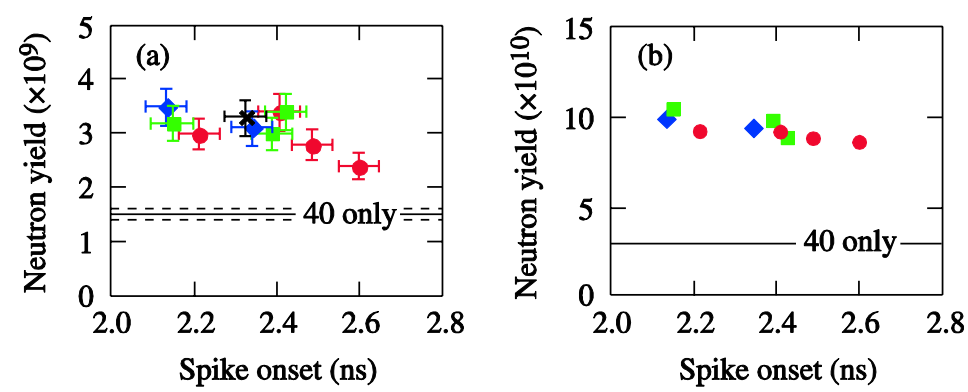

$$
\begin{aligned}
& \sim 0.9 \times 10^{15} \mathrm{~W} / \mathrm{cm}^{2} \\
& \sim 1.5 \times 10^{15} \mathrm{~W} / \mathrm{cm}^{2} \\
\times & \sim 1.5 \times 10^{15} \mathrm{~W} / \mathrm{cm}^{2}(\mathrm{IDI}-300) \\
- & \sim 3.0 \times 10^{15} \mathrm{~W} / \mathrm{cm}^{2}
\end{aligned}
$$

Figure 3. Measured (a) and 1-D-simulated (b) neutron yields in $40+20$-beam implosions for various spike onset times and spike intensities.

The effect on the measured (a) and simulated (b) neutron yield is shown in Figure 3 for various spike onset times. The different symbols represent various focus conditions. Implosions with only 40 drive beams produced $\sim 1.5 \times 10^{9}$ neutrons [solid line in Figure 3(a)]. Adding the 20 spike beams enhanced the yield by a factor of up to 2.3, with a trend of lower yields at later times. There is no significant dependence on intensity while the total energy is kept constant. A similar trend is observed in the predicted neutron yield [Figure 3(b)]. The enhancement in the simulation is up to a factor of $\sim 3.5$ when adding the spike beams because of the extra energy delivered to the target. The yield-over-clean ratio, defined as the measured to predicted neutron number, is about $3 \%$ to $5 \%$.

The hard $\mathrm{x}$-ray detector provides information on the hot-electron number and temperature. The hot-electron temperature was determined by fitting estimated values from the convolution of an exponentially decaying hard x-ray spectrum with the sensitivity of the different channels to the measurements in the four channels [18]. The inferred temperature was $\sim 35 \mathrm{keV}$ for all the shots without phase plates in the 20 spike beams, independent of laser intensity. A slightly higher temperature of $\sim 45 \mathrm{keV}$ was measured with DPP's in the 20 spike beams.

The plasma reflectivity was measured for various laser intensities. Figure 4 shows the measured fraction of laser backscatter energy of one shock beam for various laser intensities as a function of the spike onset. No significant variation is observed with spike onset. The lowest reflectivity $(\sim 6 \%)$ was measured with DPP. Without DPP, reflectivity increases from $\sim 8 \%$ at $\sim 0.8 \times 10^{15} \mathrm{~W} / \mathrm{cm}^{2}$ to $\sim 35 \%$ at $\sim 8 \times 10^{15} \mathrm{~W} / \mathrm{cm}^{2}$. The simultaneously measured back-reflection through a neighbouring drive-beam port remained constant at the level of implosions without the 20 spike beams for all beam delays and lens positions. This shows that the light from the spike beams was scattered back in a narrow cone and that there was no significant spillover into adjacent ports. 


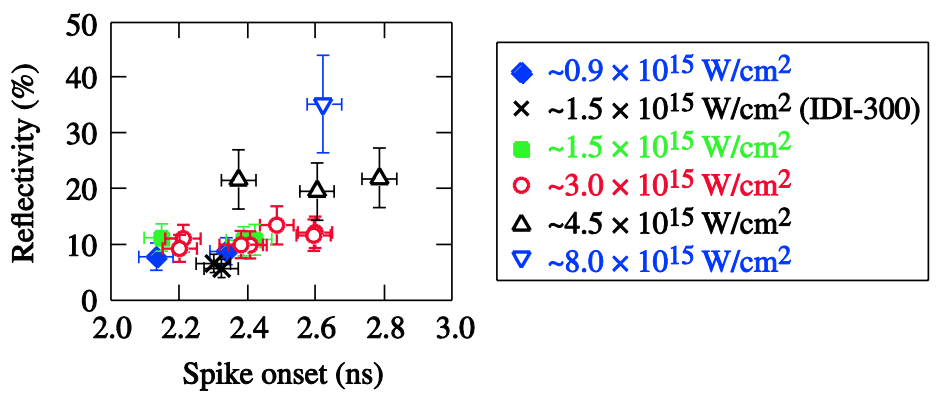

Figure 4. Percentage of the laser light (SRS + SBS) reflected back into the solid angle of the focusing lens for various intensities and delay times.

In conclusion, shock-ignition laser-plasma experiments in spherical geometry have been performed with nominal laser intensities of up to $\sim 8 \times 10^{15} \mathrm{~W} / \mathrm{cm}^{2}$. A significant amount (up to $35 \%$ ) of laser energy from the high-intensity beams was backscattered. A hot-electron-energy distribution was generated with a temperature of $\sim 40 \mathrm{keV}$, independent of laser intensity. A low hot-electron temperature is beneficial for shock ignition since these electrons are stopped in a thin outer layer of the imploding target, augmenting the strong hydrodynamic shock.

This work was supported by the U.S. Department of Energy Office of Inertial Confinement Fusion under Cooperative Agreement No. DE-FC52-08NA28302, the Fusion Science Center for Extreme States of Matter DE-FC02-04ER54789, the University of Rochester, and the New York State Energy Research and Development Authority. The support of DOE does not constitute an endorsement by DOE of the views expressed in this article.

\section{References}

[1] R. Betti et al., Phys. Rev. Lett. 98, 155001 (2007)

[2] X. Ribeyre et al., Plasma Phys. Control. Fusion 50, 025007 (2008)

[3] A. J. Schmitt et al., Fusion Sci. Technol. 56, 377 (2009)

[4] E. I. Moses, J. Phys., Conf. Ser. 112, 012003 (2008)

[5] L. J. Perkins et al., Phys. Rev. Lett. 103, 045004 (2009)

[6] J. D. Lindl, Inertial confinement fusion: The quest for ignition and energy gain using indirect drive (Springer-Verlag, New York, 1998)

[7] W. L. Kruer, The physics of laser-plasma interactions, Frontiers in physics, Vol. 73, edited by D. Pines (Addison-Wesley, Redwood City, CA, 1988)

[8] R. Betti et al., J. Phys., Conf. Ser. 112, 022024 (2008)

[9] W. Theobald et al., Phys. Plasmas 15, 056306 (2008)

[10] T. R. Boehly et al., Opt. Commun. 133, 495 (1997)

[11] W. Theobald et al., Phys. Plasmas 19, 102706 (2012)

[12] T. R. Boehly et al., J. Appl. Phys. 85, 3444 (1999)

[13] Y. Lin, T.J. Kessler, G.N. Lawrence, Opt. Lett. 21, 1703 (1996)

[14] S. P. Regan et al., J. Phys., Conf. Ser. 112, 022077 (2008)

[15] J. Delettrez et al., Phys. Rev. A 36, 3926 (1987)

[16] V. Yu. Glebov et al., Rev. Sci. Instrum. 72, 824 (2001)

[17] W. Seka et al., Phys. Plasmas 15, 056312 (2008)

[18] C. Stoeckl et al., Rev. Sci. Instrum. 72, 1197 (2001)

[19] F. H. Séguin et al., Rev. Sci. Instrum. 74, 975 (2003) 